\title{
An almost existence theorem for non-contractible periodic orbits in cotangent bundles
}

\author{
Pedro A. S. Salomão
}

Instituto de Matemática e Estatística, Universidade de São Paulo

Rua do Matão 1010, Cidade Universitária, CEP 05508-090, São Paulo-SP, Brasil

\section{Joa Weber}

Instituto de Matemática, Estatística e Computação Scientífica,

Universidade Estadual de Campinas,

Rua Sérgio Buarque de Holanda 651, Cidade Universitária "Zeferino Vaz",

CEP 13083-859, Campinas-SP, Brasil

\begin{abstract}
Assume $M$ is a closed connected smooth manifold and $H: T^{*} M \rightarrow \mathbb{R}$ a smooth proper function bounded from below. Suppose the sublevel set $\{H<d\}$ contains the zero section $M$ and $\alpha$ is a non-trivial homotopy class of free loops in $M$. Then for almost every $s \in[d, \infty)$ the level set $\{H=s\}$ carries a periodic orbit $z$ of the Hamiltonian system $\left(T^{*} M, \omega_{0}, H\right)$ representing $\alpha$. Examples show that the condition $\{H<d\} \supset M$ is necessary and almost existence cannot be improved to everywhere existence.
\end{abstract}

\section{Introduction and main result}

Suppose $M$ is a smooth manifold and its cotangent bundle $\pi: T^{*} M \rightarrow M$ is equipped with the canonical symplectic structure $\omega_{0}=-d \theta$. Here $\theta$ $(=p d q)$ denotes the canonical Liouville 1 -form on $T^{*} M$. We view the elements of $T^{*} M$ as pairs $(q, p)$ where $q \in M$ and $p \in T_{q}^{*} M$. Given any function $H$ on $T^{*} M$, the identity $d H=\omega_{0}\left(X_{H}, \cdot\right)$ uniquely determines the Hamiltonian vector field $X_{H}$ on $T^{*} M$. The integral curves of $X_{H}$ are called (Hamiltonian) orbits. They preserve the level sets of the total energy $H$. Of particular interest are periodic orbits, namely orbits $\gamma: \mathbb{R} \rightarrow T^{*} M$ such that $\gamma(t+T)=\gamma(t)$ for some constant $T>0$ and every $t \in \mathbb{R}$. The

2010 Mathematics Subject Classification. 37-06 (Primary), 70H12 (Secondary).

Key words: Hamiltonian dynamics. 
infimum ${ }^{1}$ over such $T$ is called the period of $\gamma$. Given a family of energy levels, the question arises which levels carry a periodic orbit.

Existence of a periodic orbit on a dense set of energy levels was proved for $T^{*} \mathbb{R}^{n}$ by Hofer and Zehnder [9] in 1987 and for $T^{*} M$ by Hofer and Viterbo [8] in 1988. The result for $T^{*} \mathbb{R}^{n}$ was extended to existence almost everywhere by Struwe [15] in 1990. Existence of non-contractible periodic orbits was studied among others in 1997 by Cieliebak [2] on starshaped levels in $T^{*} M$, in 2000 by Gatien and Lalonde [3] employing Lagrangian submanifolds, and in 2003 by Biran, Polterovich, and Salamon [1] on $T^{*} M$ for $M=\mathbb{R}^{n} / \mathbb{Z}^{n}$ or $M$ closed and negatively curved. The dense existence theorem in [1] was generalized in 2006 to all closed Riemannian manifolds in [17]. Theorem A below is the corresponding almost existence theorem. In contrast the almost existence theorem of Macarini and Schlenk [13] requires finiteness of the $\pi_{1}$-sensitive Hofer-Zehnder capacity. An assumption that has been verified to the best of our knowledge only for such cotangent bundles which carry certain circle actions; see [11,12]. For further references concerning dense and almost existence results we refer to [5] and concerning non-contractible orbits to [7].

Theorem A (Almost existence). Assume $M$ is a closed connected smooth manifold and $H: T^{*} M \rightarrow \mathbb{R}$ is a proper ${ }^{2}$ smooth function bounded from below. Suppose the sublevel set $\{H<d\}$ contains $M$. Then for every nontrivial homotopy class $\alpha$ of free loops in $M$ the following is true. For almost every $s \in[d, \infty)$ the level set $\{H=s\}$ carries a periodic Hamiltonian orbit $z$ that represents $\alpha$ in the sense that $[\pi \circ z]=\alpha$ where $\pi: T^{*} M \rightarrow M$ is the projection map.

Proof. There are three main ingredients in the proof. The main player is the Biran-Polterovich-Salamon (BPS) [1] capacity $\mathrm{c}_{\mathrm{BPS}}$ whose monotonicity axiom Proposition 2.3 naturally leads to the monotone function $c_{\alpha}:[d, \infty) \rightarrow[0, \infty]$ defined by

$$
c_{\alpha}(s):=c_{\mathrm{BPS}}(\{H<s\}, M ; \alpha) .
$$

Secondly, the existence result $[17$, Thm. A] concerning periodic orbits enters as follows: A priori the range of $\mathrm{c}_{\mathrm{BPS}}$ includes $\infty$ (by Definition 2.2 this is the case if no 1-periodic orbit representing $\alpha$ exists). To prove finiteness of the function $c_{\alpha}$ pick as an auxiliary quantity a Riemannian metric $g$ on $M$. Then using [17, Thm. A] one readily calculates that the BPS capacity of the open unit disk cotangent bundle relative to its zero section is equal to the smallest length $\ell_{\alpha}$ among all closed geodesics representing $\alpha$; see [17, Thm. 4.3]. The rescaling argument in Lemma 2.4 shows that

\footnotetext{
${ }^{1}$ Here and thoughout we use the convention inf $\emptyset=\infty$.

${ }^{2} \mathrm{~A}$ map is called proper if preimages of compact sets are compact.
} 


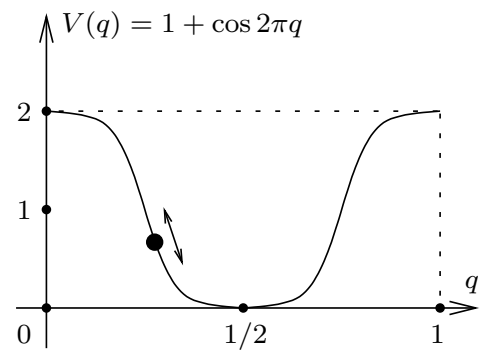

Figure 1. Potential energy $V$

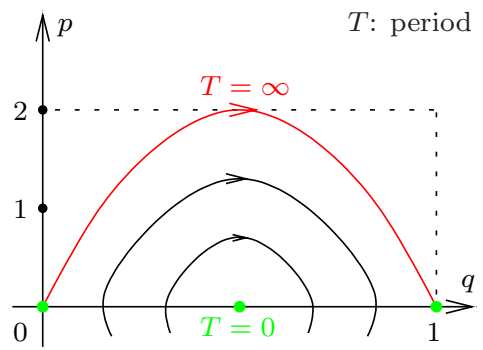

Figure 2. Pendulum phase portrait

the capacity of the open radius $r$ disk cotangent bundle $D_{r} T^{*} M$ is $r \ell_{\alpha}$. Observe that $\{H \leq s\}$ is compact since $H$ is proper and bounded below. Hence the set $\{H<s\}$ is bounded and therefore contained in $D_{r} T^{*} M$ for some sufficiently large radius $r=r(s)$. Thus $c_{\alpha}(s) \leq r(s) \ell_{\alpha}$ by the monotonicity axiom and this proves finiteness of $c_{\alpha}$.

Thirdly, by Lebesgue's last theorem, see e.g. [14], it is well known, yet amazing, that monotonicity of the map $c_{\alpha}:[d, \infty) \rightarrow[0, \infty)$ implies differentiability, thus Lipschitz continuity, at almost every point $s$ in the sense of measure theory. Now the key input is Theorem 3.1 whose proof is by an analogue of the Hofer-Zehnder method [10, Sec. 4.2] and which detects for each such $s$ a periodic orbit on the corresponding level set $\{H=s\}$.

Example 1.1 (Necessary condition). The condition $\{H<d\} \supset M$ cannot be dropped in Theorem A. First of all, together with $H$ being proper and bounded below, it guarantees that each level set $\{H=s\}$ is actually nonempty whenever $s \in[d, \infty)$. Now consider a pendulum. It moves on $M=S^{1}=\mathbb{R} / \mathbb{Z}$ in a potential of the form $V(q)=1+\cos 2 \pi q$; see Figure 1 . The Hamiltonian $H: T^{*} M=S^{1} \times \mathbb{R} \rightarrow \mathbb{R}$ is given by $H(q, p)=\frac{1}{2} p^{2}+V(q)$; see Figure 2 for the phase portrait. Energies below the maximum value 2 of the potential $V$ do not allow for full rotations. For such low energies the pendulum can just swing hence and forth. Observe that $\{H<1\} \not \supset M$. On the other hand, for any energy $s \in[1,2)$ the level set $\{H=s\}$ consists of a periodic orbit which is contractible onto the stable (lower) equilibrium point $(x, y) \equiv(1 / 2,0)$. So none of these orbits represents a homotopy class $\alpha \neq 0$. (For $s>2$ the sets $\{H=s\}$ represent classes $\alpha \neq 0$. The set $\{H=2\}$ consists of the unstable (upper) equilibrium point and two homoclinic orbits one of them indicated red in Figure 2.) 


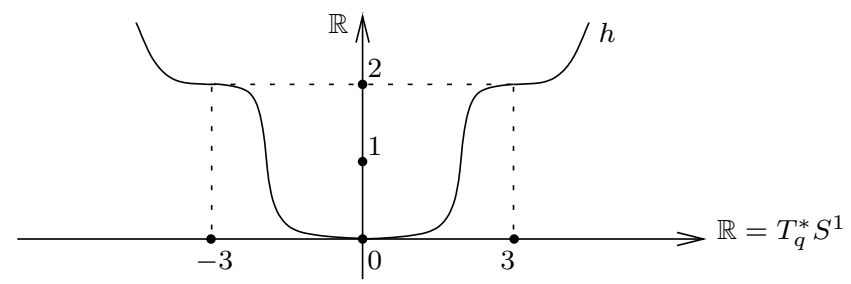

Figure 3. Hamiltonian $H=h(p)$ without non-constant orbits on $\{H=2\}$

Example 1.2 (Existence everywhere not true). To see that almost existence in Theorem A cannot be improved to everywhere existence consider the case $M=S^{1}$ and a Hamiltonian $H: S^{1} \times \mathbb{R} \rightarrow \mathbb{R}$ of the form $H(q, p)=h(p)$. More precisely, pick a proper smooth function $h \geq 0$ with $h(0)=0$ and $h( \pm 3)=2$ and where the points $0, \pm 3$ are the only points of slope zero; see Figure 3. Then $\{H<1\}$ contains $M=S^{1}$. Moreover, the whole level set $\{H=2\}$ consists of critical points of $H$. Therefore on $\{H=2\}$ the Hamiltonian vector field $X_{H}$ vanishes identically and so all orbits are necessarily constant.

In contrast to this critical level counterexample it should be interesting to find a regular level of a smooth Hamiltonian $H$ as in Theorem A without a periodic orbit in a given homotopy class $\alpha \neq 0$. One possible way to achieve this is to start with an energy level with finitely many periodic orbits representing $\alpha$, then destroy them using the symplectic plugs constructed in [4].

For general symplectic manifolds existence may fail completely; see [18] and [16] for examples of closed symplectic manifolds admitting Hamiltonians with no non-constant periodic orbits.

\section{Symplectic capacities}

To fix notation consider $\mathbb{R}^{2 n}$ with coordinates $\left(x_{1}, \ldots, x_{n}, y_{1}, \ldots, y_{n}\right)$ and symplectic form $\omega_{0}=\sum_{i=1}^{n} d x_{i} \wedge d y_{i}$. Associate to each symplectic manifold $(N, \omega)$, of fixed dimension $2 n>0$ and possibly with boundary, a number $c(N, \omega) \in[0, \infty]$ that satisfies the axioms:

- Monotonicity: $c\left(N_{1}, \omega_{1}\right) \leq c\left(N_{2}, \omega\right)$ whenever there is a symplectic embedding $\psi:\left(N_{1}, \omega_{1}\right) \rightarrow\left(N_{2}, \omega_{2}\right)$.

- Conformality: $c(N, \lambda \omega)=|\lambda| c(N, \omega), \forall \lambda \in \mathbb{R} \backslash\{0\}$.

- Non-Triviality: $c\left(B(1), \omega_{0}\right)=c\left(Z(1), \omega_{0}\right)=\pi$. 
Here $B(r)=\left\{(x, y) \in \mathbb{R}^{2 n}:|x|^{2}+|y|^{2}<r^{2}\right\}$ is the ball of radius $r>0$ and $Z(r)=\left\{(x, y) \in \mathbb{R}^{2 n}: x_{1}^{2}+y_{1}^{2}<r^{2}\right\}$ is the symplectic cylinder of radius $r>0$. On $\left(\mathbb{R}^{2 n}, \omega_{0}\right)$, one checks the following re-scaling property:

$$
U \subset \mathbb{R}^{2 n} \text { open } \Rightarrow c\left(\lambda U, \omega_{0}\right)=\lambda^{2} c\left(U, \omega_{0}\right), \forall \lambda \in \mathbb{R} \backslash\{0\} .
$$

A map $(N, \omega) \mapsto c(N, \omega)$ satisfying the three axioms above is called a symplectic capacity. Gromov introduced this notion in [6] and showed that

$$
c_{0}(N, \omega):=\sup \left\{\pi r^{2}: \exists \text { symplectic embedding } \psi:\left(B(r), \omega_{0}\right) \rightarrow(N, \omega)\right\}
$$

is a symplectic capacity, called Gromov's width. It satisfies $c_{0}(N, \omega) \leq$ $c(N, \omega)$ for any other symplectic capacity $c$. One of the consequences of the existence of a symplectic capacity is the non-squeezing theorem which asserts that

$\exists$ symplectic embedding $\psi:\left(B(r), \omega_{0}\right) \rightarrow\left(Z(R), \omega_{0}\right) \quad \Leftrightarrow \quad r \leq R$.

2.1. Hofer-Zehnder capacity. Hofer and Zehnder introduced in [10] a symplectic capacity defined in terms of the Hamiltonian dynamics on the underlying symplectic manifold $(N, \omega)$. Recall that a smooth function $H$ : $N \rightarrow \mathbb{R}$ determines the Hamiltonian vector field $X_{H}$ by $i_{X_{H}} \omega=d H$. We say that a periodic orbit of $\dot{x}=X_{H}(x)$ is fast if its period is $<1$. A function $H: N \rightarrow \mathbb{R}$ is called admissible if it admits a maximum and the following conditions hold:

- $0 \leq H \leq \max H<\infty$.

- $\exists \bar{K} \subset \bar{N} \backslash \partial N$ compact, such that $\left.H\right|_{N \backslash K}=\max H$.

- $\exists U \subset N$ open and non-empty, such that $\left.H\right|_{U}=0$.

- $\dot{x}=X_{H} \circ x$ admits no non-constant fast periodic orbits.

The set of admissible Hamiltonians is denoted by $\mathcal{H}_{a}(N, \omega)$. Let

$$
\mathrm{c}_{\mathrm{HZ}}(N, \omega):=\sup \left\{\max H \mid H \in \mathcal{H}_{a}(N, \omega)\right\} .
$$

Theorem 2.1 (Hofer-Zehnder). $\mathrm{c}_{\mathrm{HZ}}$ is a symplectic capacity.

We should remark that the hard part of proving Theorem 2.1 is to show that $\mathrm{c}_{\mathrm{HZ}}$ satisfies the non-triviality axiom.

2.2. BPS relative capacity. Fix a closed manifold $M$. The components $\mathcal{L}_{\alpha} M$ of the free loop space $\mathcal{L} M:=C^{\infty}\left(S^{1}, M\right)$ are labelled by the elements $\alpha=[\gamma]$ of the set $\tilde{\pi}_{1}(M)$ of homotopy classes of free loops $\gamma$ in $M$. Here and throughout we identify $S^{1}$ with $\mathbb{R} / \mathbb{Z}$ and think of $\gamma$ as a smooth map $\gamma: \mathbb{R} \rightarrow M$ that satisfies $\gamma(t+1)=\gamma(t)$ for every $t \in \mathbb{R}$. A function $H \in C_{0}^{\infty}\left(S^{1} \times T^{*} M\right)$ determines a 1-periodic family of compactly supported vector fields $X_{H_{t}}$ on $T^{*} M$ by $d H_{t}=\omega_{0}\left(X_{H_{t}}, \cdot\right)$. Let

$$
\mathcal{P}_{1}(H ; \alpha):=\left\{z: S^{1} \rightarrow T^{*} M \mid \dot{z}(t)=X_{H_{t}}(z(t)) \forall t \in S^{1},[\pi \circ z]=\alpha\right\}
$$


be the set of 1-periodic orbits of $X_{H_{t}}$ whose projections to $M$ represent $\alpha$.

Definition 2.2. Following [1] assume $W \subset T^{*} M$ is an open subset which contains the zero section $M$. For any constant $b>0$ consider the set

$$
\mathcal{H}_{b}(W):=\left\{H \in C_{0}^{\infty}\left(S^{1} \times W\right) \mid m_{0}(H):=\max _{S^{1} \times M} H \leq-b\right\} .
$$

The BPS capacity of $W$ relative $M$ and with respect to $\alpha \in \tilde{\pi}_{1}(M)$ is defined by

$$
\operatorname{c}_{\mathrm{BPS}}(W, M ; \alpha):=\inf \left\{b>0 \mid \mathcal{P}_{1}(H ; \alpha) \neq \emptyset \text { for every } H \in \mathcal{H}_{b}(W)\right\} .
$$

Note that $c_{B P S}$ takes values in $[0, \infty]$ since we use the convention inf $\emptyset=$ $\infty$. Furthermore, the BPS capacity is a relative symplectic capacity.

Proposition 2.3 (Monotonicity [1, Prop. 3.3.1]). If $W_{1} \subset W_{2} \subset T^{*} M$ are open subsets containing $M$ and $\alpha \in \tilde{\pi}_{1}(M)$, then $\mathrm{c}_{\mathrm{BPS}}\left(W_{1}, M ; \alpha\right) \leq$ $\mathrm{c}_{\mathrm{BPS}}\left(W_{2}, M ; \alpha\right)$.

Fix a Riemannian metric on $M$ and constants $r, b>0$. Denote by $D T^{*} M$ the open unit disk cotangent bundle and by $D_{r} T^{*} M$ the one of radius $r$. Observe that

$$
H \in \mathcal{H}_{b}\left(D T^{*} M\right) \quad \Longleftrightarrow \quad H_{r} \in \mathcal{H}_{r b}\left(D_{r} T^{*} M\right)
$$

whenever the Hamiltonians $H$ and $H_{r}$ are related by $H_{r}(t, q, p)=r$. $H\left(t, q, \frac{p}{r}\right)$. In addition, pick $\alpha \in \tilde{\pi}_{1}(M)$. Then there is the crucial bijection

$$
\mathcal{P}_{1}(H ; \alpha) \rightarrow \mathcal{P}_{1}\left(H_{r} ; \alpha\right):(x, y) \mapsto(x, r y)
$$

asserting that the 1-periodic orbits of $H$ correspond naturally with those of $H_{r}$.

Lemma 2.4 (Rescaling). $\mathrm{c}_{\mathrm{BPS}}\left(D_{r} T^{*} M, M ; \alpha\right)=r \cdot \mathrm{c}_{\mathrm{BPS}}\left(D T^{*} M, M ; \alpha\right)$.

Proof. By definition (2) of the BPS capacity we obtain that

$$
\begin{aligned}
& \mathrm{c}_{\mathrm{BPS}}\left(D_{r} T^{*} M, M ; \alpha\right) \\
& =\inf \left\{r b>0 \mid \mathcal{P}_{1}\left(H_{r} ; \alpha\right) \neq \emptyset \text { for every } H_{r} \in \mathcal{H}_{r b}\left(D_{r} T^{*} M\right)\right\} \\
& =r \cdot \inf \left\{b>0 \mid \mathcal{P}_{1}(H ; \alpha) \neq \emptyset \text { for every } H \in \mathcal{H}_{b}\left(D T^{*} M\right)\right\} \\
& =r \cdot \operatorname{cBPS}_{\mathrm{BPS}}\left(D T^{*} M, M ; \alpha\right)
\end{aligned}
$$

where the second step uses (3) and (4).

Corollary 2.5. $c_{\mathrm{BPS}}\left(D_{r} T^{*} M, M ; \alpha\right)=r \ell_{\alpha}$ where $\ell_{\alpha}$ is the smallest length among all closed curves representing $\alpha$.

Proof. $\mathrm{c}_{\mathrm{BPS}}\left(D T^{*} M, M ; \alpha\right)=\ell_{\alpha}$ by [17, Thm. 4.3]. Apply Lemma 2.4. 
An almost existence theorem for non-contractible periodic orbits in cotangent bundles 391

\section{The Hofer-Zehnder method}

Assume the Hamiltonian $H: T^{*} M \rightarrow \mathbb{R}$ is smooth, proper, and bounded from below and a sublevel set $\{H<d\}$ contains $M$. Fix a non-trivial homotopy class $\alpha$ of free loops in $M$. Consider the monotone function $c_{\alpha}$ defined on the interval $[d, \infty)$ by (1). By Lebesgue's last theorem, see e.g. [14, p. 401], the function $c_{\alpha}$ is differentiable at almost every point in the sense of measure theory.

Theorem 3.1. Assume $s_{0} \in[d, \infty)$ is a regular value of $H$ and $c_{\alpha}$ is Lipschitz continuous at $s_{0}$. Then the hypersurface $S=H^{-1}\left(s_{0}\right)$ carries a periodic orbit $z_{T}$ of $X_{H}$ that represents $\alpha$ and where $T>0$ is the period.

Proof. The proof is an adaption of the Hofer-Zehnder method [10, Sec. 4.2] to the case at hand. To emphasize this we mainly keep their notation. Fix $s_{0}$ as in the hypothesis of the theorem. Then $S_{0}:=H^{-1}\left(s_{0}\right)$ is a hypersurface ${ }^{3}$ in $T^{*} M$ by the inverse function theorem. It is compact since $H$ is proper and it bounds the open set $\dot{B}_{0}:=\left\{H<s_{0}\right\}$ since $H$ is bounded below. Furthermore, by the implicit function theorem and compactness of $S_{0}$ there is a constant $\mu>0$ such that $s_{0}+\varepsilon$ is a regular value of $H$ and $S_{\varepsilon}:=H^{-1}\left(s_{0}+\varepsilon\right)$ is diffeomorphic to $S_{0}$ whenever $\varepsilon \in[-\mu, \mu]$. Note that $S_{\varepsilon}$ bounds the open set $\dot{B}_{\varepsilon}:=\left\{H<s_{0}+\varepsilon\right\}$ which itself contains the zero section $M$ of $T^{*} M$. Furthermore, since $c_{\alpha}$ is Lipschitz continuous at $s_{0}$ there is a constant $L>0$ such that

$$
c(\varepsilon)-c(0) \leq L \varepsilon, \quad c(\varepsilon):=c_{\alpha}\left(s_{0}+\varepsilon\right),
$$

for every $\varepsilon \in[-\mu, \mu]$; otherwise, choose $\mu>0$ smaller. We proceed in three steps I-III.

I. Pick $\tau \in(0, \mu)$. Then there is a Hamiltonian $K \in C_{0}^{\infty}\left(S^{1} \times \dot{B}_{0}\right)$ whose maximum over the zero section satisfies

$$
-c(0)<m_{0}(K) \leq-(c(0)-L \tau)
$$

and which does not admit any 1-periodic orbit representing $\alpha$. Indeed if no such $K$ exists, then $\mathrm{c}_{\mathrm{BPS}}\left(\dot{B}_{0}, M ; \alpha\right) \leq c(0)-L \tau$ by definition $(2)$ of the BPS capacity. But $c(0)=c_{\alpha}\left(s_{0}\right)=\mathrm{c}_{\mathrm{BPS}}\left(\dot{B}_{0}, M ; \alpha\right)$ and we obtain the contradiction $c(0) \leq c(0)-L \tau$. Now pick a smooth function $f: \mathbb{R} \rightarrow$ $[-3 L \tau, 0]$ such that

$$
\begin{array}{lll}
f(s)=-3 L \tau & \text { if } & s \leq 0 \\
f(s)=0 & \text { if } & s \geq \frac{\tau}{2} \\
0<f^{\prime}(s) \leq 7 L & \text { if } & 0<s<\frac{\tau}{2}
\end{array}
$$

\footnotetext{
${ }^{3}$ A hypersurface is a smooth submanifold of codimension 1.
} 


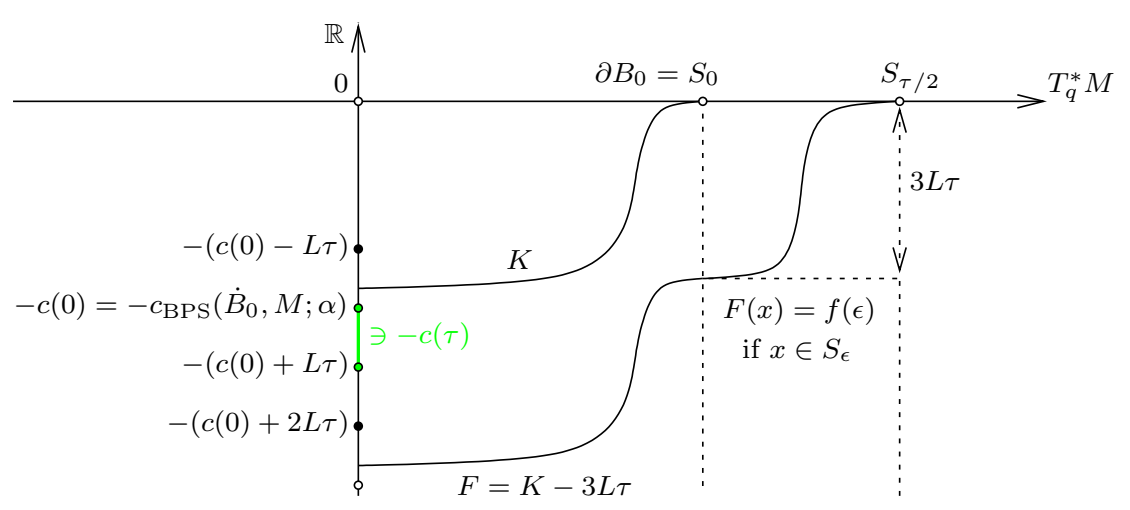

FiguRe 4. Hamiltonians $F \in \mathcal{H}_{c(\tau)}\left(\dot{B}_{\tau}\right)$ and $K$ with $\mathcal{P}_{1}(K ; \alpha)=\emptyset$

and consider the Hamiltonian $F \in C_{0}^{\infty}\left(S^{1} \times \dot{B}_{\tau}\right)$ defined by

$$
\begin{array}{lll}
F(x)=K(x)-3 L \tau & \text { if } & x \in \dot{B}_{0} \\
F(x)=f(\varepsilon) & \text { if } & x \in S_{\varepsilon}=H^{-1}\left(s_{0}+\varepsilon\right), 0 \leq \varepsilon<\tau \\
F(x)=0 & \text { if } & x \notin \dot{B}_{\tau}
\end{array}
$$

and illustrated by Figure 4. By (5) the Hamiltonian $F$ satisfies the estimate

$$
m_{0}(F)=m_{0}(K)-3 L \tau \leq-(c(0)-L \tau)-3 L \tau<-(c(0)+L \tau) \leq-c(\tau) .
$$

Since $m_{0}(F) \leq-\mathrm{c}_{\mathrm{BPS}}\left(\dot{B}_{\tau}, M ; \alpha\right)$ the definition (2) of the BPS capacity shows that the set $\mathcal{P}_{1}(F ; \alpha)$ is not empty. In other words, there is a 1periodic orbit $z$ of $X_{F}$ that represents $\alpha$. Observe that $z$ cannot intersect $\dot{B}_{0}$ : Due to compact support the open set $\dot{B}_{0}$ is invariant under the flow of $K$. But the flows of $K$ and $K-3 L \tau=F$ coincide. Thus, if $z$ intersects $\dot{B}_{0}$, then it stays completely inside. But this is impossible since $\mathcal{P}_{1}(K ; \alpha)=\emptyset$. On the other hand, since $\alpha \neq 0$ the orbit $z$ of $X_{F}$ is non-constant and therefore it must intersect the regions foliated by the hypersurfaces $S_{\varepsilon}$ where $0<\varepsilon<\frac{\tau}{2}$. But each of them is a level set of $F$, hence invariant under the flow of $X_{F}$. This shows that $z$ lies on $S_{\varepsilon}$ for some $0<\varepsilon<\frac{\tau}{2}$.

II. Repeat the argument for each element of a sequence $\tau_{j} \rightarrow 0$ to obtain sequences $F_{j}$ and $\varepsilon_{j}$ and a sequence $z_{j}$ of 1-periodic orbits of $X_{F_{j}}$ that lie on $S_{\varepsilon_{j}}$ and where $\varepsilon_{j} \rightarrow 0$. Next we interpret each $z_{j}$ as a $T_{j}$-periodic orbit of $X_{H}$ by rescaling time. Most importantly, the periods $T_{j}$ are uniformly 
bounded from above by $7 L$. To see this note that on the open set

$$
U:=\bigcup_{\varepsilon \in(-\mu, \mu)} S_{\varepsilon}=\bigcup_{\varepsilon \in(-\mu, \mu)} H^{-1}\left(s_{0}+\varepsilon\right)
$$

the Hamiltonian $H$ is obviously given by $H(x)=s_{0}+\varepsilon$ whenever $x \in S_{\varepsilon}$. For each $\tau_{j}$ and each $\varepsilon \in\left[0, \tau_{j}\right)$ we have

$$
F_{j}(x)=f_{j}\left(H(x)-s_{0}\right)=f_{j}(\varepsilon)
$$

for every $x \in S_{\varepsilon}$. At such $x$ use the definition of $X_{F_{j}}$ and the chain rule to get

$$
\omega_{0}\left(X_{F_{j}}, \cdot\right)=d F_{j}=f_{j}^{\prime}\left(H-s_{0}\right) d H=\omega_{0}\left(f_{j}^{\prime}(\varepsilon) X_{H}, \cdot\right) .
$$

Thus, because $z_{j}$ lies on $S_{\varepsilon_{j}}$, it satisfies the equation

$$
\dot{z}_{j}(t)=X_{F_{j}} \circ z_{j}(t)=T_{j} \cdot X_{H} \circ z_{j}(t), \quad T_{j}:=f_{j}^{\prime}\left(\varepsilon_{j}\right),
$$

and the periodic boundary condition $z_{j}(t+1)=z_{j}(t)$ for every $t \in \mathbb{R}$.

III. Uniform boundedness of the periods $T_{j}$ is crucial in the following proof of existence of a 1-periodic orbit $z$ of $X_{H}$ which lies on the original level hypersurface $S_{0}=H^{-1}\left(s_{0}\right)$ and represents the given class $\alpha$. Indeed note that $S_{\varepsilon_{j}} \subset\left\{H \leq s_{0}+\mu\right\}=: B_{\mu}$ and that $B_{\mu}$ is compact since $H$ is proper and bounded below. In other words, the sequence of loops $z_{j}$ is uniformly bounded in $C^{0}$. Concerning $C^{1}$ we obtain the uniform estimate

$$
\left|\dot{z}_{j}(t)\right|=\left|T_{j}\right| \cdot\left|X_{H} \circ z_{j}(t)\right| \leq 7 L\left\|X_{H}\right\|_{C^{0}\left(B_{\mu}\right)}
$$

for all $t \in S^{1}$ and $j \in \mathbb{N}$. Therefore by the Arzelà-Ascoli theorem there is a subsequence, still denoted by $z_{j}$, which converges in $C^{0}$ and by using the equation for $z_{j}$ even in $C^{\infty}$ to a smooth 1-periodic solution $z$ of the equation $\dot{z}=T \cdot X_{H}(z)$ where $T=\lim _{j \rightarrow \infty} T_{j}$. Since $\varepsilon_{j} \rightarrow 0$ the orbit $z$ takes values on the desired level hypersurface $S_{0}=H^{-1}\left(s_{0}\right)$. To prove that $z=(x, y)$ represents the same class $\alpha$ as does each $z_{j}=\left(x_{j}, y_{j}\right)$ we need to show that $[x]=\left[x_{j}\right]$ for some $j$. To see this consider the injectivity radius $\iota>0$ of the compact Riemannian manifold $(M, g)$ and pick $j$ sufficiently large such that the Riemannian distance between $x(t)$ and $x_{j}(t)$ is less than $\iota / 2$ for every $t \in S^{1}$. Setting $\exp _{x(t)} \xi(t)=x_{j}(t)$ provides the desired homotopy $h_{\lambda}(t)=\exp _{x(t)} \lambda \xi(t)$ between $h_{0}=x$ and $h_{1}=x_{j}$.

Reparametrize time to obtain the $T$-periodic solution $z_{T}(t):=z(t / T)$ of

$$
\dot{z}_{T}(t)=\frac{1}{T} \dot{z}(t / T)=X_{H} \circ z(t / T)=X_{H} \circ z_{T}(t)
$$

which obviously represents the same class $\alpha$ as $z$. Since $\alpha \neq 0$ the loop $z_{T}$ cannot be constant and so the period necessarily satisfies $T>0$. This concludes the proof of Theorem 3.1. 
FIRST AUTHOR. Financial support: FAPESP grant 2011/16265-8 and CNPq grant 303651/2010-5. Email: psalomao@gmail.com

SECOND AUTHOR. Financial support: FAPESP grant 2011/01830-1. Email: joa@math.sunysb.edu

\section{References}

[1] P. Biran, L. Polterovich, and D. Salamon. Propagation in Hamiltonian dynamics and relative symplectic homology. Duke Math. J., 119(1):65-118, 2003.

[2] K. Cieliebak. Symplectic boundaries: Creating and destroying closed characteristics. Geom. Funct. Anal., 7(2):269-321, 1997.

[3] D. Gatien and F. Lalonde. Holomorphic cylinders with Lagrangian boundaries and Hamiltonian dynamics. Duke Math. J., 102(3):485-511, 2000.

[4] V.L. Ginzburg. A smooth counterexample to the Hamiltonian Seifert conjecture in $\mathbb{R}^{6}$. Int. Math. Res. Not., 1997(13):641-650, 1997.

[5] V.L. Ginzburg. The Weinstein conjecture and theorems of nearby and almost existence. In Jerrold E. Marsden and Tudor S. Ratiu, editors, The Breadth of Symplectic and Poisson Geometry, volume 232 of Progress in Mathematics, pages 139-172. Birkhäuser Boston, 2005.

[6] M. Gromov. Pseudo holomorphic curves in symplectic manifolds. Invent. Math., 82:307-347, 1985.

[7] B.Z. Gürel. On Non-contractible Periodic Orbits of Hamiltonian Diffeomorphisms. ArXiv e-prints, 1210.3866:1-9, October 2012.

[8] H. Hofer and C. Viterbo. The Weinstein conjecture in cotangent bundles and related results. Ann. Sc. Norm. Super. Pisa, Cl. Sci., IV. Ser., 15(3):411-445, 1988.

[9] H. Hofer and E. Zehnder. Periodic solutions on hypersurfaces and a result by C. Viterbo. Invent. Math., 90:1-9, 1987.

[10] H. Hofer and E. Zehnder. Symplectic invariants and Hamiltonian dynamics. Birkhäuser Advanced Texts. Basel: Birkhäuser. xiii, 341 p., 1994.

[11] K. Irie. Hofer-Zehnder capacity and a Hamiltonian circle action with noncontractible orbits. ArXiv e-prints, 1112.5247:1-6, December 2011.

[12] L. Macarini. Hofer-Zehnder capacity and Hamiltonian circle actions. Commun. Contemp. Math., 6(6):913-945, 2004.

[13] L. Macarini and F. Schlenk. A refinement of the Hofer-Zehnder theorem on the existence of closed characteristics near a hypersurface. Bull. Lond. Math. Soc., 37(2):297-300, 2005.

[14] C.C. Pugh. Real mathematical analysis. Undergraduate Texts in Mathematics. New York, NY: Springer. xi, 437 p. , 2002.

[15] M. Struwe. Existence of periodic solutions of Hamiltonian systems on almost every energy surface. Bulletin of the Brazilian Mathematical Society, 20(2):4958, 1990.

[16] M. Usher. Many closed symplectic manifolds have infinite Hofer-Zehnder capacity. ArXiv e-prints, 1101.4986:1-31, January 2011.

[17] J. Weber. Noncontractible periodic orbits in cotangent bundles and Floer homology. Duke Math. J., 133(3):527-568, 2006.

[18] E. Zehnder. Remarks on periodic solutions on hypersurfaces. Periodic solutions of Hamiltonian systems and related topics, Proc. NATO Adv. Res. Workshop, Il Ciocco/Italy 1986, NATO ASI, Ser. C, 209:267-279, 1987. 